\title{
Evaluation of the brief Wisconsin Inventory of smoking dependence motives in African-American and European-American heavy smokers
}

\author{
Jennie Z. Ma ${ }^{1}{ }^{*}$, Ming D. Li $^{2}$ and Thomas J. Payne ${ }^{3}$ \\ ${ }^{1}$ Department of Public Health Sciences, University of Virginia, Charlottesville, VA, USA \\ ${ }^{2}$ Department of Psychiatry and Neurobehavioral Sciences, University of Virginia, Charlottesville, VA, USA \\ ${ }^{3}$ Department of Otolaryngology and Communicative Sciences, University of Mississippi Medical Center, Jackson, MS, USA
}

\section{Edited by:}

Marco Diana, University of Sassari, Italy

Reviewed by:

Jennifer G. Plebani, University of Pennsylvania School of Medicine, USA

Shaolin Wang, University of Virginia, USA

*Correspondence:

Jennie Z. Ma, Department of Public Health Sciences, University of Virginia, PO Box 800717,

Charlottesville, VA 22908, USA.

e-mail: jzm4h@virginia.edu
Introduction: Emerging evidence indicates that nicotine dependence should be measured multidimensionally. A brief version of the Wisconsin Inventory of Smoking Dependence Motives (WISDM) has recently been developed in which the item count has been reduced from 68 to 37. The objectives of this study were to replicate the initial findings in a larger heavy-smoking sample, and determine whether the WISDM structure is applicable to both African-American (AA) and European-American (EA) heavy smokers. Methods: Smokers were selected from our Mid-South Tobacco Family and Case-Control studies. Available data from 2,522 smokers was selected, involving 1,633 AA and 889 EA participants. Both exploratory and confirmatory analyses were employed to evaluate the psychometric characteristics of the Brief WISDM. Results: AAs and EAs were similar in age, sex, education, marital status, cigarettes per day, and Fagerström Test for Nicotine Dependence score. Internal consistency evaluations for Brief WISDM subscales were adequate but generally lower than that of the full-scale version. Confirmatory factor analyses did not yield desirable fits for AA or EA smokers. Exploratory factor analysis revealed good agreement for item loadings on the four primary dependence motives subscales (Automaticity, Loss of Control, Craving, and Tolerance) but discrepancies on all secondary dependence motives subscales except Taste/Sensory Processes. Specific item loadings for subscales differed by ethnicity. Conclusion: The Brief WISDM demonstrated reasonable psychometric properties in our large sample. Together, we provide support for the general validity of the brief form but suggest individual item selections may benefit from further investigation.

Keywords: nicotine dependence, smoking motives, WISDM

\section{INTRODUCTION}

Tobacco use and cigarette smoking in particular remains a leading preventable cause of morbidity and death. Despite considerable progress in the public health arena in recent years, about 45.3 million individuals smoke (CDC, 2011), with an estimated annual cost of $\$ 193$ billion in the USA due to premature death and disability (CDC, 2008). Approximately 443,000 deaths each year are attributed to smoking, which is the most common cause of cancer-related deaths in the USA, including deaths from lung cancer, laryngeal cancer, esophageal cancer, oral cancer, and bladder cancer (CDC, 2007). Tobacco use is also a leading cause of heart disease, stroke, and chronic obstructive pulmonary disease (CDC, 2007).

Traditionally, nicotine dependence (ND) has been characterized by the development of tolerance with regular use and the emergence of withdrawal symptoms as a function of abstention/reduction. This construct has important implications for understanding the long-term use of tobacco, as well as the difficulty in achieving and maintaining abstinence when attempting to quit. One of the most widely used measures of ND in both clinical and research settings is the Fagerström Test for Nicotine Dependence (FTND) (Heatherton et al., 1991).

Emerging evidence indicates that ND is a complex disorder having physiological, psychological (behavioral, cognitive, affective), and social components. The Wisconsin Inventory of Smoking Dependence Motives (WISDM-68) is comprised of 68 items within 13 subscales designed to measure multidimensional motivational influences that drive tobacco use (Piper et al., 2004): Affiliative Attachment, Automaticity, Loss of Control, Behavioral Choice/Melioration, Cognitive Enhancement, Craving, Cue Exposure/Associative Processes, Negative Reinforcement, Positive Reinforcement, Social/Environmental Goads, Taste/Sensory Properties, Tolerance, and Weight Control. Item selection was based on both empirical findings and theories of substance dependence (Piper et al., 2004, 2008). The breadth of the dimensions permits a more comprehensive evaluation of ND compared with other instruments such as FTND. The WISDM-68 has demonstrated good psychometric characteristics (Piper et al., 2008; Shenassa et al., 2009). To reduce assessment burden, the Brief WISDM was developed (Smith et al., 2010), retaining 37 items 
which load onto 11 subscales. On the basis of three samples of adult smokers with sizes of 366, 608, and 393 including 10.4, 22.1, and 100\% African-American (AA) smokers, respectively, the authors concluded that the Brief WISDM is reliable and valid, and thus suitable for use in place of the original version.

Over the last 10 years, we have recruited more than 4,000 $\mathrm{AA}$ and EA smokers into our Mid-South Tobacco Case Control (MSTCC) and Mid-South Tobacco Family (MSTF) cohort studies, with the majority being heavy smokers. Our work (Li et al., 2005; Ma et al., 2005; Beuten et al., 2006) and that of others (Perez-Stable et al., 1998; Payne and Diefenbach, 2003; Fernander et al., 2008; Saccone et al., 2009) has identified differences across ethnicity with respect to genetic factors, physiologic processes, and some behavioral differences that are associated with ND. Thus, it is of interest to see whether the WISDM is of similar relevance across those populations. For the current study, our primary objectives were to (a) evaluate and attempt to replicate Smith et al.'s (2010) findings regarding the psychometric properties of the Brief WISDM in this large sample of smokers, as well as (b) to determine whether the Brief WISDM structure can be validated independently in both AA and EA smokers.

\section{MATERIALS AND METHODS STUDY POPULATION}

Smokers were selected from two genetic studies on ND, the MSTF (1999-2004) and MSTCC (2005-present) studies. Participants were of low to middle SES status, recruited primarily from community-based sites and outpatient medical facilities in the states of Tennessee, Mississippi, Arkansas, and Texas. Informed consent was obtained from all participants in accordance with approved procedures from institutional review boards.

The MSTF is a family-based study designed to identify susceptibility loci and genes for ND, enrolling 2,037 participants from 200 EA and 402 AA families. Detailed information on recruitment and characteristics of study participants has been previously reported (Li et al., 2006, 2008). A heavy smoker proband was recruited first who was at least 18 years old, had smoked for at least 5 years, and had consumed an average of 20 cigarettes per day (CPD) or more for the last 12 months. Once a proband was identified, biological parents and additional siblings were recruited whenever possible. If biological parents were not available, we attempted to recruit at least three full siblings per family. The MSTCC Study is an ongoing case-control genetic study of ND in which both nonsmokers and heavy smokers were recruited. Smokers had smoked for at least 5 years with an average of at least $20 \mathrm{CPD}$ for the past 12 months at the time of recruitment. Because of concerns regarding familial clustering, we selected only unrelated smokers from the MSTF sample by including both smoking parents (as they are not biologically related to each other), or a smoker (parent or offspring) randomly selected per family. While the possibility that shared environment might account for some correlated effects, our preliminary analyses indicated this only applied to less than $2 \%$ of participants, and thus is unlikely to be a significant factor. These unrelated smokers from the MSTF study and all smokers from the MSTCC study comprised the study sample used for this report.

\section{NICOTINE DEPENDENCE MEASURES}

The ND measures used in this study were the FTND (Heatherton et al., 1991), CPD, and the WISDM-68 (Piper et al., 2008). The item set of the WISDM-68 includes those of the Brief WISDM (Smith et al., 2010), and subscales from both instruments were calculated. This replicates the approach used by Smith et al. (2010).

We also examined the WISDM primary dependence motives (PDM) and secondary dependence motives (SDM) scales, which were developed from latent class analyses and factor mixture models (Piper et al., 2008). The PDM was calculated as the mean of the Automaticity, Loss of Control, Craving, and Tolerance scores. It indexes heavy-smoking characterized by frequent, strong, and bothersome craving. In contrast, the SDM scale consists of the remaining WISDM subscales, assessing diverse motives that reflect instrumental and contextually bound effects of smoking.

The Brief WISDM retained the top four items loading on each of the PDM subscales, and the top three items for the SDM subscales of the WISDM-68. Negative Reinforcement and Positive Reinforcement subscales were consolidated into Affective Enhancement, and the Behavioral Choice/Melioration subscale was dropped from the Brief WISDM.

\section{STATISTICAL ANALYSIS}

Internal consistency

We first evaluated the internal consistency of each Brief WISDM subscale, with a Cronbach's $\alpha$ of 0.7 considered to reflect good reliability (Cronbach, 1951). For comparison, we also calculated Cronbach's $\alpha$ for the WISDM-68 subscales. The analyses were carried out for the total sample, as well as for AA and EA smokers separately.

\section{Concurrent validity}

We assessed the concurrent validity of the Brief WISDM based on correlations with FTND and CPD following the approach used by Smith et al. (2010). The differences between AA and EA smokers in the correlations of WISDM subscales with FTND/CPD were tested using the Fisher's $z$-transformation, available in the PROC CORR procedure of SAS (2008). The overall association of the Brief WISDM with the FTND was evaluated in a multiple linear regression analysis.

\section{Confirmatory and exploratory factor analysis}

We first performed confirmatory factor analysis (CFA) to evaluate whether the 11-factor model of the Brief WISDM identified by Smith et al. (2010) fit our data. As an additional test of stability and validation, we evaluated the fit of each Brief WISDM subscale using an exploratory structural equation modeling (ESEM) approach (Asparouhov and Muthen, 2009). Model fit indices for CFA and ESEM analyses included the Akaike information criterion (AIC), Bayesian information criterion (BIC), standardized root mean square residual (SRMR), comparative fit index (CFI), Tucker-Lewis index (TLI), and root mean square error of approximation (RMSEA). The AIC and BIC were used for general evaluation, whereas the other indices were evaluated according to the recommendations of $\mathrm{Hu}$ and Bentler (1999), such that a SRMR value $<0.09$, CFI and TLI values between 0.90 and 0.95 , and RMSEA values between 0.06 and 0.08 were indicative of acceptable fit. Following the ESEM analyses, we further performed the 
exploratory factor analyses (EFA) for each of the Brief WISDM subscales to determine whether a better fit model could be derived. The items with high item-to-total correlations and factor loadings were selected, four items for PDM subscales and three for SDM subscales.

The descriptive and correlational analyses were performed using SAS 9.2 (SAS, 2008). Modeling analyses such as EFA, ESEM, and CFA were performed using Mplus version 6 (Muthen and Muthen, 1998-2010).

\section{RESULTS}

\section{SAMPLE CHARACTERISTICS}

A total of 2,522 smokers were selected from our MSTCC and MSTF databases, of which 1,633 were AAs and 889 were EAs. Table 1 displays demographics and smoking characteristics for AA and EA smokers, both separately and together. The AA and EA smokers were similar with respect to age $(43.1 \pm 11.8$ versus $42.3 \pm 11.4$ ), sex (\% female: 50.5 versus 52.5 ), education (high school or higher: 77.3 versus $79.1 \%$ ), and marital status (married: 37.3 versus $41.2 \%$ ), respectively.

On average, AA smokers smoked 1.3 fewer CPD than EA smokers $(26.4 \pm 9.6$ versus $27.7 \pm 9.1)$, but achieved higher scores on the FTND $(8.1 \pm 1.8$ versus $7.7 \pm 2.1)$. This is consistent with previous literature indicating that, equated for intake, AAs often appear more dependent on nicotine per self-report or plasma cotinine measurement (Ahijevych et al., 1996, 2002; Ahijevych and Parsley, 1999). For the smoking motives, AA smokers scored equally or slightly lower than EA smokers on 11 of 13 subscales of the WISDM-68 (Table 1).

\section{INTERNAL CONSISTENCY}

All the Brief WISDM and WISDM-68 subscales revealed Cronbach's $\alpha>0.70$ except for the Brief WISDM Cue Exposure $(\alpha=0.67)$ in EA smokers (Table 2$)$. These coefficients were similar to those reported by Smith et al. (2010) for the Brief WISDM subscales. In general, the coefficients for the Brief WISDM subscales were modestly smaller than the corresponding WISDM-68 subscales. Indeed, PDM subscale (Automaticity, Loss of Control, Craving, and Tolerance) values were similar as well, whereas some of the SDM subscale values were considerably lower for the Brief WISDM than the corresponding WISDM-68 subscales. The largest discrepancy was found for Cue Exposure/Associative Processes, where $\alpha=0.74$ and 0.67 for the Brief WISDM compared with 0.84 and 0.82 for the WISDM-68 in AA and EA smokers, respectively. This may be due, in part, to the fact that Brief WISDM subscales are based on fewer items overall (four items in PDM subscales and three items in SDM subscales).

\section{CONCURRENT VALIDITY}

We evaluated concurrent validity by examining the correlations between the Brief WISDM and two other common measures of

Table 1 | Smoker's descriptive characteristics and WISDM-68 Scores.

\begin{tabular}{|c|c|c|c|}
\hline Measure & EA smokers ( $N=889)$ & AA smokers $(N=1633)$ & All smokers $(\mathrm{N}=\mathbf{2 5 2 2})$ \\
\hline Gender (\% female) & 52.5 & 50.5 & 51.2 \\
\hline Ethnicity (\% AA) & 0 & 100 & 64.8 \\
\hline Education (\% $\geq$ high school) & 79.1 & 77.3 & 77.9 \\
\hline$\%$ Married & 41.2 & 37.3 & 38.7 \\
\hline Age & $42.3 \pm 11.4$ & $43.1 \pm 11.8$ & $42.8 \pm 11.7$ \\
\hline Cigarettes per day & $27.7 \pm 9.1$ & $26.4 \pm 9.6$ & $26.9 \pm 9.4$ \\
\hline FTND score & $7.7 \pm 2.1$ & $8.1 \pm 1.8$ & $7.9 \pm 1.9$ \\
\hline \multicolumn{4}{|l|}{ WISDM-68 SCALES } \\
\hline Automaticity & $4.4 \pm 1.3(4.4,3.8-5.0)$ & $4.2 \pm 1.2(4.4,4.0-4.8)$ & $4.3 \pm 1.2(4.4,4.0-4.8)$ \\
\hline Craving & $4.7 \pm 1.3(4.8,4.4-5.5)$ & $4.4 \pm 1.2(4.5,4.0-5.0)$ & $4.5 \pm 1.2(4.5,4.0-5.0)$ \\
\hline Loss of control & $4.6 \pm 1.3(4.8,4.0-5.3)$ & $4.4 \pm 1.2(4.5,4.0-5.0)$ & $4.5 \pm 1.3(4.5,4.0-5.0)$ \\
\hline Tolerance & $4.7 \pm 1.2(4.6,4.2-5.4)$ & $4.4 \pm 1.1(4.4,4.0-5.0)$ & $4.5 \pm 1.2(4.6,4.0-5.0)$ \\
\hline Affiliative attachment & $4.3 \pm 1.5(4.6,3.4-5.2)$ & $4.4 \pm 1.3(4.8,4.2-5.0)$ & $4.4 \pm 1.4(4.8,4.0-5.2)$ \\
\hline Behavioral choice/melioration & $4.4 \pm 1.3(4.6,3.9-5.1)$ & $4.3 \pm 1.2(4.6,4.1-4.9)$ & $4.4 \pm 1.2(4.6,4.0-5.0)$ \\
\hline Cognitive enhancement & $4.2 \pm 1.5(4.4,3.6-5.0)$ & $4.2 \pm 1.2(4.4,3.8-4.8)$ & $4.2 \pm 1.3(4.4,3.8-5.0)$ \\
\hline Cue exposure/assoc processes & $4.6 \pm 1.1(4.7,4.1-5.3)$ & $4.4 \pm 1.1(4.6,4.0-5.0)$ & $4.5 \pm 1.1(4.6,4.1-5.0)$ \\
\hline Negative reinforcement & $4.7 \pm 1.2(4.8,4.3-5.3)$ & $4.5 \pm 1.1(4.7,4.3-5.0)$ & $4.6 \pm 1.2(4.8,4.3-5.2)$ \\
\hline Positive reinforcement & $4.5 \pm 1.3(4.6,4.0-5.2)$ & $4.4 \pm 1.2(4.6,4.0-5.0)$ & $4.5 \pm 1.2(4.6,4.0-5.0)$ \\
\hline Social/environmental goads & $4.7 \pm 1.4(4.8,4.0-5.8)$ & $4.5 \pm 1.2(4.5,4.0-5.0)$ & $4.6 \pm 1.3(4.5,4.0-5.3)$ \\
\hline Taste/sensory properties & $4.6 \pm 1.3(4.7,4.2-5.3)$ & $4.6 \pm 1.1(4.7,4.3-5.0)$ & $4.6 \pm 1.2(4.7,4.2-5.2)$ \\
\hline Weight control & $3.8 \pm 1.6(4.4,2.4-4.8)$ & $4.1 \pm 1.3(4.4,3.8-4.8)$ & $4.0 \pm 1.4(4.4,3.4-4.8)$ \\
\hline PDM & $4.6 \pm 1.1(4.6,4.2-5.1)$ & $4.4 \pm 1.0(4.5,4.1-4.8)$ & $4.4 \pm 1.1(4.5,4.1-4.9)$ \\
\hline SDM & $4.4 \pm 1.0(4.6,4.0-4.9)$ & $4.4 \pm 1.0(4.6,4.2-4.8)$ & $4.4 \pm 1.0(4.6,4.1-4.9)$ \\
\hline WISDM total & $58.3 \pm 13.3(60,53-64)$ & $56.9 \pm 13.0(59,54-63)$ & $57.4 \pm 13.1(59,54-63)$ \\
\hline
\end{tabular}

EA, European-American; AA, African-American; entries are mean \pm SD (median, lower to upper quartile) for WISDM measures; PDM, primary dependence motives; SDM, secondary dependence motives. 
Table 2 | Internal consistency of WISDM-68 and brief WISDM subscales.

\begin{tabular}{|c|c|c|c|c|c|c|}
\hline \multirow[t]{2}{*}{ WISDM subscale } & \multicolumn{2}{|c|}{ EA smokers ( $N=889)$} & \multicolumn{2}{|c|}{ AA smokers $(N=1,633)$} & \multicolumn{2}{|c|}{ All smokers $(N=2,522)$} \\
\hline & WISDM-68 & Brief WISDM & WISDM-68 & Brief WISDM & WISDM-68 & Brief WISDM \\
\hline Automaticity & 0.86 & 0.85 & 0.85 & 0.83 & 0.85 & 0.84 \\
\hline Loss of control & 0.77 & 0.77 & 0.75 & 0.75 & 0.76 & 0.76 \\
\hline Craving & 0.86 & 0.86 & 0.81 & 0.81 & 0.83 & 0.83 \\
\hline Tolerance & 0.74 & 0.73 & 0.74 & 0.72 & 0.74 & 0.73 \\
\hline Affiliative attachment & 0.90 & 0.87 & 0.89 & 0.82 & 0.90 & 0.84 \\
\hline Behavioral choice/melioration ${ }^{a}$ & 0.87 & - & 0.88 & - & 0.88 & - \\
\hline Cognitive enhancement & 0.91 & 0.87 & 0.86 & 0.81 & 0.88 & 0.83 \\
\hline Cue exposure/assoc processes & 0.82 & 0.67 & 0.84 & 0.74 & 0.84 & 0.71 \\
\hline Affective enhancement ${ }^{b}$ & - & 0.79 & - & 0.76 & - & 0.78 \\
\hline Negative reinforcement & 0.86 & - & 0.86 & - & 0.86 & - \\
\hline Positive reinforcement & 0.86 & - & 0.85 & - & 0.85 & - \\
\hline Social/environmental goads & 0.88 & 0.86 & 0.83 & 0.79 & 0.86 & 0.82 \\
\hline Taste/sensory properties & 0.88 & 0.87 & 0.85 & 0.82 & 0.86 & 0.84 \\
\hline Weight control & 0.91 & 0.88 & 0.88 & 0.84 & 0.90 & 0.86 \\
\hline PDM & 0.89 & 0.88 & 0.91 & 0.91 & 0.90 & 0.90 \\
\hline SDM & 0.91 & 0.86 & 0.95 & 0.92 & 0.94 & 0.89 \\
\hline WISDM total & 0.97 & 0.95 & 0.98 & 0.96 & 0.98 & 0.96 \\
\hline
\end{tabular}

${ }^{a}$ This subscale was not included in Brief WISDM; ${ }^{b}$ New scale for Brief WISDM, derived from original positive and negative reinforcement.

ND: FTND and CPD. The 11 Brief WISDM subscales displayed significant correlations with FTND and CPD (Table 3), but generally were lower than those reported by Smith et al. (2010). Contrary to expectations, PDM subscales did not display stronger correlations with the FTND than SDM subscales. The correlations between the Brief WISDM subscales and the FTND were consistently higher for AA smokers than EA smokers, except for the Tolerance subscale. Correlations with the FTND were significantly different between AA and EA smokers for Automaticity, Loss of Control, Craving, Affiliative Attachment, Cue Exposure/Associative, Social/Environmental Goads, and Taste/Sensory Properties subscales. In contrast, Brief WISDM subscale correlations with CPD across AA and EA smokers were different only for the Tolerance subscale. For comparison, we evaluated the concurrent validity of the WISDM-68 with the FTND and CPD and found the magnitude of those associations to be similar to those for the Brief WISDM. Similar differences in the pattern of correlations with FTND/CPD were found for the WSIDM-68 subscales.

The overall association of the Brief WISDM with the FTND was evaluated by entering all 11 Brief WISDM subscales into a multiple linear regression analysis. Automaticity, Loss of Control, Affiliative Attachment, Social/Environmental Goads, and Weight Control subscales were significantly associated with the FTND for AA and EA smokers; Craving and Tolerance subscales were associated for EA smokers only.

\section{CONFIRMATORY FACTOR ANALYSIS}

We performed CFA separately for AA and EA smokers to evaluate the 11-factor model of the Brief WISDM, based on the final model derived by Smith et al. (2010), which freely estimates correlated errors for four pairs of items identified from their earlier analyses (based on a personal communication with
Dr. Smith) and from our samples. These four pairs of items were: (1) "I usually want to smoke right after I wake up" and "I smoke within the first $30 \mathrm{~min}$ of awakening in the morning," (2) "cigarettes control me" and "sometimes I feel like cigarettes rule my life," (3) "my smoking is out of control" and "I consider myself a heavy smoker," and (4) "other smokers would consider me a heavy smoker" and "I consider myself a heavy smoker." The CFAs yielded the following results: $\chi^{2}(\mathrm{df}=570)=5569.2$, $\mathrm{SRMR}=0.052, \mathrm{CFI}=0.855, \mathrm{TLI}=0.831$, and $\mathrm{RMSEA}=0.077$ [95\% confidence interval $(\mathrm{CI})=0.075,0.079]$ for AA smokers and $\chi^{2}(\mathrm{df}=570)=2521.6, \mathrm{SRMR}=0.055, \mathrm{CFI}=0.897, \mathrm{TLI}=0.879$, and $\mathrm{RMSEA}=0.064(95 \% \mathrm{CI}=0.061,0.066)$ for EA smokers. These fit indices deliver a mixed message, as the 11-factor model fit seemed to fit the data reasonably well based on the SRMR and RMSEA, but not as well as by CFI and TLI for both AA and EA smokers.

\section{EVALUATION OF ITEM SELECTION}

Given the mixed results for the 11-factor model fit in AA and EA smokers, we expanded our evaluation of the Brief WISDM by examining the fit of individual Brief WISDM subscales using an exploratory SEM approach and by assessing the items selected for each subscale with EFA. The exploratory SEM results indicate that most subscales indeed fit reasonably well with the corresponding items for both AA and EA smokers. Specifically, CFI and TLI were consistently $\geq 0.90$ and SRMR $<0.09$ for most subscales for both AA and EA smokers, except for Loss of Control (TLI = 0.88) in AA smokers, and Tolerance in both AA and EA smokers. However, RMSEA was inconsistent across the 11 subscales, which was $>0.08$ for the Loss of Control and Affective Enhancement in AA smokers and for Social/Environmental Goads, Taste/Sensory Properties, and Affiliative Attachment subscales in EA smokers. The poorest fit 
Table 3 | Correlations between WISDM-68 and Brief WISDM subscales and with validity measures.

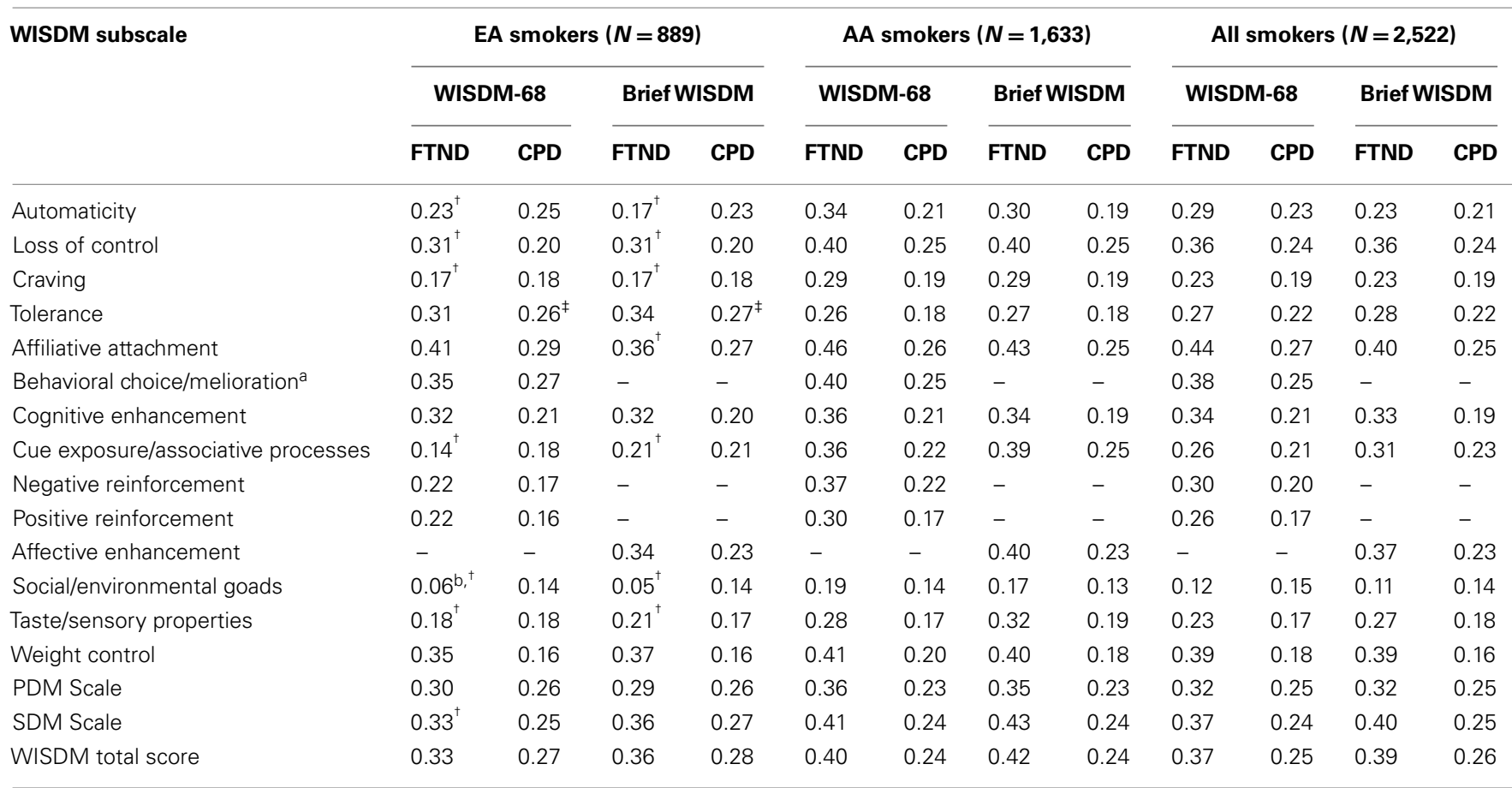

${ }^{a}$ This subscale was not included in Brief WISDM; ${ }^{b}$ not significant at 0.05 level.

${ }^{+}$Significant differences in the correlations with FTND between AA and EA smokers;

${ }^{\ddagger}$ Significant differences in the correlations with CPD between AA and EA Smokers.

was found in the Tolerance subscale for both AA and EA smokers (AAs: $\mathrm{CFI}=0.914, \mathrm{TLI}=0.828, \mathrm{SRMR}=0.051, \mathrm{RMSEA}=0.136$; EAs: $\mathrm{CFI}=0.806, \mathrm{TLI}=0.611, \mathrm{SRMR}=0.078, \mathrm{RMSEA}=0.208)$.

For the Brief WISDM, four items were selected for each of the PDM subscales and three items for the SDM subscales. When assessing the EFA results for our data based on the item-to-total correlations and factor loadings, we found that virtually the same sets of items would be selected for the PDM subscales for AA and EA smokers (Table 4). However, substantial discrepancies were found for the SDM subscales in which alternative items could be selected on the basis of their relative contributions (factor loadings and/or item-to-total correlations) to the corresponding subscales. The last two columns of Table 4 summarize the numbers of items selected by our analyses in contrast with those represented in the Brief WISDM as it currently stands. The largest discrepancy was found in the Affective Enhancement subscale, where only one of the three items agreed for AA smokers and none agreed for EA smokers.

\section{DISCUSSION}

We endeavored to replicate and extend the recently published findings for the Brief WISDM using our sample comprised of AA and EA heavy smokers recruited from the Mid-South region of the US. In general, the AA and EA smokers in our sample had basic demographic characteristics similar to those of the Smith et al. (2010) sample. As expected, our samples revealed higher levels of ND based on the CPD, FTND, and to some degree, WISDM68 scales. The Brief WISDM subscales demonstrated good internal
Table 4 | Evaluation of the items tapped by the Brief WISDM subscales.

\begin{tabular}{|c|c|c|c|c|}
\hline \multirow[t]{2}{*}{ Items } & \multirow{2}{*}{ 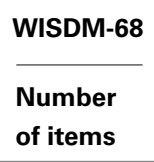 } & \multirow{2}{*}{$\begin{array}{l}\text { Brief WISDM } \\
\text { Number } \\
\text { of items }\end{array}$} & \multicolumn{2}{|c|}{ Agreements $^{\mathrm{a}}$} \\
\hline & & & EA & AA \\
\hline Automaticity & 5 & 4 & 4 & 3 \\
\hline Loss of control & 4 & 4 & 4 & 4 \\
\hline Craving & 4 & 4 & 4 & 4 \\
\hline Tolerance & 5 & 4 & 4 & 4 \\
\hline $\begin{array}{l}\text { Taste/sensory } \\
\text { processes }\end{array}$ & 6 & 3 & 3 & 3 \\
\hline Cognitive enhancement & 5 & 3 & 2 & 2 \\
\hline $\begin{array}{l}\text { Social/environmental } \\
\text { goads }\end{array}$ & 4 & 3 & 2 & 2 \\
\hline Weight control & 5 & 3 & 2 & 2 \\
\hline $\begin{array}{l}\text { Cue exposure/ } \\
\text { associative processes }\end{array}$ & 7 & 3 & 1 & 2 \\
\hline Affiliative attachment & 12 & 3 & 2 & 1 \\
\hline Affective enhancement & 11 & 3 & 0 & 1 \\
\hline Total \# of items & 68 & 37 & 28 & 28 \\
\hline
\end{tabular}

${ }^{a}$ Number of matches between items selected by analyses and those currently assigned to Brief WISDM.

consistency, although the Cue Exposure/Associative Processes subscale was marginal, and the Cronbach $\alpha$ was lower overall than reported by Smith et al. (2010). Adequate concurrent validity was 
demonstrated as well, although correlations with the FTND and CPD were lower than previously reported.

The findings from our CFA suggest a less desirable fit, and EFA revealed mixed results. We found good agreement for items loading onto the four PDM subscales (Automaticity, Loss of Control, Craving, and Tolerance) but considerable discrepancies on all SDM subscales except Taste/Sensory Processes. Two of three items for the Cognitive Enhancement, Social/Environmental Goads, and Weight Control subscales were in agreement, and only one of three items was in agreement for Cue Exposure/Associative Processes and Affiliative Attachment. For the Affective Enhancement subscale, one item was in agreement for AA smokers and none for EA smokers. In general, this may suggest that the SDM scales have poorer stability for higher nicotine-dependent individuals. Whether this is a function of subscale structure, or more fundamentally the validity of the items for this subset of the population awaits further investigation. Replication of the current findings represents one approach to establishing the applicability of these results. Examining the relationships between Brief WISDM subscales and key tobacco variables in high versus low nicotine-dependent smokers may also yield useful information.

Our sample consisted of individuals who are heavy smokers. Although the average age was similar to that in the validation sample, members of our sample smoked more CPD on average (6 more for AAs and 10 more for EAs compared to samples used in the Smith et al., 2010 study). Accordingly, the FTND scores were higher than those in the original sample. This could partially explain the less-than-desirable fit of the Brief WISDM and some of the discrepancies regarding individual item loadings, although it should be noted that discrepant findings were not evident across all subscales. The truncated range of daily smoking rate limits the generalizability of our sample to the general smoking population, but its strength is that these individuals may be reflective of the more highly dependent population of smokers who are more likely to seek treatment (Irvin and Brandon, 2000). As higher-rate smokers have a higher risk for the development and exacerbation of tobacco-related diseases (USDHHS, 2004), we believe an in-depth evaluation of this group is justified and highly significant. In addition, our group has published numerous papers on the genetic contributions to ND in this important sample, both relative to non-smokers and when comparing across ethnicity, providing further evidence of its importance and suitability of this population as a target for study (Ma et al., 2005, 2010; Li, 2006, 2008).

Separate evaluation of AA and EA smokers indicated that with the exception of Cue Exposure/Associative Processes, internal consistencies were somewhat higher for EA smokers, whereas measures of concurrent validity were relatively better for AA smokers. Interestingly, the Craving and Tolerance subscales were associated with the FTND only for EA smokers, suggesting those subscales

\section{REFERENCES}

Ahijevych, K., Gillespie, J., Demirci, M., and Jagadeesh, J. (1996). Menthol and nonmenthol cigarettes and smoke exposure in black and white women. Pharmacol. Biochem. Behav. 53, 355-360.

Ahijevych, K., and Parsley, L. A. (1999). Smoke constituent exposure and stage of change in black and white women cigarette smokers. Addict. Behav. 24, 115-120.

Ahijevych, K. L., Tyndale, R. F., Dhatt, R. K., Weed, H. G., and Browning, K. K. (2002). Factors influencing cotinine half-life during smoking abstinence in African American and

do not measure the same factors in AA and EA smokers. Previous research has demonstrated that AAs generally present as more nicotine-dependent than EAs, perhaps due in part to metabolism rate differences for nicotine (Perez-Stable et al., 1998; Berg et al., 2010). It is also possible that the WISDM/Brief WISDM is sensitive to these differences, thus yielding a different relationship to the FTND across ethnicity. Such findings may ultimately lead to a more sensitive, targeted assessment of these factors.

The 11-factor model fit better for EA than AA smokers, and specific item loadings for each subscale differed across ethnicity. One potential explanation is that a larger proportion of EA smokers comprised the original sample for scale development $(58.6 \%$ EA in the original sample compared with $35.2 \%$ in the current study). This seems unlikely to be a significant factor, as our sample size was sufficiently large to detect relatively small effects. Another possibility is that the AA smokers we recruited represent a more extreme segment of the distribution of all smokers, yielding a sample whose responses are somewhat more restricted in variance, and thus less likely to reveal differences. However, inspection of Table 1 suggests this is unlikely, given that the average scores for these measures for our AA participants are actually lower, while the SD's are similar to EA smokers. It is unknown whether these findings are comparable to those of Smith et al. (2010), as they did not analyze ethnic samples separately. Regardless, the overall higher smoking rate of the current sample (both EAs and AAs) may contribute to some of the differences found, and raise the possibility that the current findings have greater relevance for those smokers at the higher end of the ND continuum. Finally, two of the three samples employed by Smith et al. (2010) were enrolled in clinical trials, whereas the current study employed only community samples. It is possible that individuals enrolled in clinical trials respond differently than those not seeking treatment.

In summary, the 37-item Brief WISDM demonstrated reasonably good internal consistency and validity in our replication sample. Discrepancies were noted across ethnicities with respect to specific item loadings, particularly for the SDM subscales, which might be explained in part by our heavy-smoking participants, and the fact that we specifically examined ethnic differences. However, the 11-factor model did not reveal a good fit; alternative items may need to be considered. It is possible that the SDM subscales are somewhat less relevant for heavier smokers.

\section{ACKNOWLEDGMENTS}

We thank all participants in the MSTCC and MSTF studies. We also thank Ms. Xing Tang for her analytical assistance in this study and Dr. David L Bronson for his editing of this paper. Funding for this project was provided in part by NIH grant DA-012844 to Ming D. Li.

Caucasian women. Nicotine Tob. Res. 4, 423-431.

Asparouhov, T., and Muthen, B. O. (2009). Exploratory structural equation modeling. Struct. Equ. Modeling 16, 397-438.

Berg, J. Z., Mason, J., Boettcher, A. J., Hatsukami, D. K., and Murphy, S. E. (2010). Nicotine metabolism in African Americans and European Americans: variation in glucuronidation by ethnicity and UGT2B10 haplotype. J. Pharmacol. Exp. Ther. 332, 202-209.

Beuten, J., Payne, T. J., Ma, J. Z., and Li, M. D. (2006). Significant association of catechol-Omethyltransferase (COMT) 
haplotypes with nicotine dependence in male and female smokers of two ethnic populations. Neuropsychopharmacology 31, 675-684.

CDC. (2007). Cigarette smoking among adults - United States, 2006. MMWR Morb. Mortal. Wkly. Rep. $56,1157-1161$.

CDC. (2008). Smoking-attributable mortality, years of potential life lost, and productivity losses - United States, 2000-2004. MMWR Morb. Mortal. Wkly. Rep. 57, 1226-1228.

CDC. (2011). Vital signs: current cigarette smoking among adults aged $>/=18$ years - United States, 2005-2010. MMWR Morb. Mortal. Wkly. Rep. 60, 1207-1212.

Cronbach, L. J. (1951). Coefficient alpha and the internal structure of tests. Psychometrika 16, 297-334.

Fernander, A. F., Schumacher, M., and Nasim, A. (2008). Sociocultural stress, smoking risk, and cessation among African American women. J. Black Psychol. 34, 49-69.

Heatherton, T. F., Kozlowski, L. T., Frecker, R. C., and Fagerstrom, K. O. (1991). The Fagerstrom Test for nicotine dependence: a revision of the Fagerstrom Tolerance Questionnaire. Br. J. Addict. 86, 1119-1127.

Hu, L., and Bentler, P. M. (1999). Cutoff criteria for fit indexes in covariance structure analysis: conventional critera versus new alternatives. Struct. Equ. Modeling 6, 1-55.

Irvin, J. E., and Brandon, T. H. (2000). The increasing recalcitrance of smokers in clinical trials. Nicotine Tob. Res. 2, 79-84.

Li, M. D. (2006). The genetics of nicotine dependence. Curr. Psychiatry Rep. 8, 158-164.

Li, M. D. (2008). Identifying susceptibility loci for nicotine dependence: 2008 update based on recent genome-wide linkage analyses. Hum. Genet. 123, 119-131.

Li, M. D., Beuten, J., Ma, J. Z., Payne, T. J., Lou, X. Y., Garcia, V., Duenes, A. S., Crews, K. M., and Elston, R. C. (2005). Ethnic- and genderspecific association of the nicotinic acetylcholine receptor alpha4 subunit gene (CHRNA4) with nicotine dependence. Hum. Mol. Genet. 14, 1211-1219.

Li, M. D., Ma, J. Z., Payne, T. J., Lou, X. Y., Zhang, D., Dupont, R. T., and Elston, R. C. (2008). Genome-wide linkage scan for nicotine dependence in European Americans and its converging results with African Americans in the Mid-South Tobacco Family sample. Mol. Psychiatry 13, 407-416.

Li, M. D., Payne, T. J., Ma, J. Z., Lou, X. Y., Zhang, D., Dupont, R. T., Crews, K. M., Somes, G., Williams, N. J., and Elston, R. C. (2006). A genomewide search finds major susceptibility Loci for nicotine dependence on chromosome 10 in African Americans. Am. J. Hum. Genet. 79, 745-751.

Ma, J. Z., Beuten, J., Payne, T. J., Dupont, R. T., Elston, R. C., and Li, M. D. (2005). Haplotype analysis indicates an association between the DOPA decarboxylase (DDC) gene and nicotine dependence. Hum. Mol. Genet. 14, 1691-1698.

Ma, J. Z., Payne, T. J., and Li, M. D. (2010). Significant association of glutamate receptor, ionotropic Nmethyl-D-aspartate 3A (GRIN3A), with nicotine dependence in European- and African-American smokers. Hum. Genet. 127, 503-512.

Muthen, L. K., and Muthen, B. O. (19982010). Mplus Users' Guide, 3rd Edn. Los Angles, CA: Muthen \& Muthen.
Payne, T. J., and Diefenbach, L. (2003). Characteristics of African American smokers: a brief review. Am. J. Med. Sci. 326, 212-215.

Perez-Stable, E. J., Herrera, B., Jacob, P. III, and Benowitz, N. L. (1998). Nicotine metabolism and intake in black and white smokers. JAMA 280, 152-156.

Piper, M. E., Bolt, D. M., Kim, S. Y., Japuntich, S. J., Smith, S. S., Niederdeppe, J., Cannon, D. S. and Baker, T. B. (2008). Refining the tobacco dependence phenotype using the Wisconsin Inventory of Smoking Dependence Motives. J. Abnorm. Psychol. 117, 747-761.

Piper, M. E., Piasecki, T. M., Federman, E. B., Bolt, D. M., Smith, S. S., Fiore, M. C., and Baker, T. B. (2004). A multiple motives approach to tobacco dependence: the Wisconsin Inventory of Smoking Dependence Motives (WISDM-68). J. Consult. Clin. Psychol. 72, 139-154.

Saccone, N. L., Wang, J. C., Breslau, N., Johnson, E. O., Hatsukami, D., Saccone, S. F., Grucza, R. A., Sun, L., Duan, W., Budde, J., Culverhouse, R. C., Fox, L., Hinrichs, A. L., Steinbach, J. H., Wu, M., Rice, J. P., Goate, A. M., and Bierut, L. J. (2009). The CHRNA5-CHRNA3CHRNB4 nicotinic receptor subunit gene cluster affects risk for nicotine dependence in African-Americans and in European-Americans. Cancer Res. 69, 6848-6856.

SAS. (2008). SAS/Genetics User's Guide. Cary, NC: SAS Institute Inc.

Shenassa, E. D., Graham, A. L., Burdzovic, J. A., and Buka, S. L. (2009). Psychometric properties of the Wisconsin Inventory of Smoking Dependence Motives (WISDM-68): a replication and extension. Nicotine Tob. Res. 11, 1002-1010.
Smith, S. S., Piper, M. E., Bolt, D. M., Fiore, M. C., Wetter, D. W., Cinciripini, P. M., and Baker, T. B. (2010). Development of the Brief Wisconsin Inventory of Smoking Dependence Motives. Nicotine Tob. Res. 12, 489-499.

U. S. Department of Health and Human Services. (2004). The Health Consequences of Smoking: A Report of the Surgeon General. Atlanta: U.S. Department of Health and Human Services, Centers for Disease Control and Prevention, National Center for Chronic Disease Prevention and Health Promotion, Office on Smoking and Health.

Conflict of Interest Statement: The authors declare that the research was conducted in the absence of any commercial or financial relationships that could be construed as a potential conflict of interest.

Received: 10 February 2012; accepted: 30 March 2012; published online: 26 April 2012.

Citation: Ma JZ, Li MD and Payne TJ (2012) Evaluation of the brief Wisconsin Inventory of smoking dependence motives in African-American and EuropeanAmerican heavy smokers. Front. Psychiatry 3:36. doi: 10.3389/fpsyt.2012.00036 This article was submitted to Frontiers in Addictive Disorders, a specialty of Frontiers in Psychiatry.

Copyright $(0) 2012 \mathrm{Ma}$, Li and Payne. This is an open-access article distributed under the terms of the Creative Commons Attribution Non Commercial License, which permits non-commercial use, distribution, and reproduction in other forums, provided the original authors and source are credited. 